\title{
Application of JAERI quantum molecular dynamics model for collisions of heavy nuclei
}

\author{
Tatsuhiko Ogawa ${ }^{1, a}$, Shintaro Hashimoto ${ }^{1}$, Tatsuhiko Sato ${ }^{1}$, and Koji Niita ${ }^{2}$ \\ ${ }^{1}$ Japan Atomic Energy Agency, 2-4 Shirakata, Tokai-mura, Naka-gun, Ibaraki-ken, Japan, 319-1195; \\ ${ }^{2}$ Research Organization for Information Science and Technology, 2-4 Shirakata, Tokai-mura, Naka-gun, \\ Ibaraki-ken, Japan, 319-1195;
}

\begin{abstract}
The quantum molecular dynamics (QMD) model incorporated into the generalpurpose radiation transport code PHITS was revised for accurate prediction of fragment yields in peripheral collisions. For more accurate simulation of peripheral collisions, stability of the nuclei at their ground state was improved and the algorithm to reject invalid events was modified. In-medium correction on nucleon-nucleon cross sections was also considered. To clarify the effect of this improvement on fragmentation of heavy nuclei, the new QMD model coupled with a statistical decay model was used to calculate fragment production cross sections of Ag and Au targets and compared with the data of earlier measurement. It is shown that the revised version can predict cross section more accurately.
\end{abstract}

\section{Introduction}

Heavy ion beam irradiation on heavy nuclei is common in the heavy ion cancer therapy and fundamental science studies on nuclear physics. Accurate prediction of reactions induced by heavy ions with general-purpose radiation transport simulation codes is important for such applications to estimate detector performance and evaluate radiological safety of the experiment. JQMD (Jaeri Quantum Molecular Dynamics code) [1] incorporated into PHITS (Particle and Heavy Ion Transport code System) [2] is one of such codes and used at some facilities. Calculation of neutron production for heavy ion irradiation was well benchmarked so far in various target-projectile-energy combinations [3, 4]. To predict fragment production from light nuclei and medium-mass nuclei, JQMD was recently upgraded to JQMD-2.0 [5]. Its performance was tested in various conditions [6, 7] and it was confirmed that fragment production owing to peripheral collisions, underestimated by previous version of JQMD, was predicted for better accuracy.

In the revision of JQMD to JQMD-2.0, stability of nuclei before reactions, description of the interactions between nucleons, and in-medium effect on nucleon-nucleon interactions were modified. However, it is likely that ground-state stability and in-medium effect in light to medium-mass nuclei and those in heavy nuclei are different. Therefore, JQMD-2.0 was applied to interactions of heavy nuclei such as Ag and Au and its accuracy was benchmarked.

ae-mail: ogawa.tatsuhiko@jaea.go.jp 


\section{Method}

Description of reaction mechanism in JQMD and JQMD-2.0 is explained briefly. Since complete description of simulation scheme in JQMD-2.0 is provided elsewhere [5], the aspects of JQMD-2.0 important for simulation of heavy nuclei are given here. By randomly choosing the coordinates of each nucleon in the 6-dimension phase space and adjusting them to reproduce the total binding energy, the initial states of the target and projectile are configured. In JQMD, thus configured ground states were directly used for collision event simulation; however, time evolution of the configured states was followed and only the states which stayed stable for $150 \mathrm{fm} / \mathrm{c}$ was used for collision simulation in JQMD-2.0. After configuring the ground states, the target and the projectile were transferred to their center-of-mass frame. In the frame, nucleons are transported for $150 \mathrm{fm} / \mathrm{c}$ based on their initial momenta and interactions between nucleons. Interaction between nucleons is one of the most important issues in JQMD because nucleon-nucleon interaction was not described in a Lorentz-covariant form in JQMD, therefore nuclei configured in their rest frame were occasionally excited or disintegrated during time evolution in the center-of-mass frame. Lorentz-covariant equation of motion adopted in JQMD-2.0 [8] is described as follows;

$$
\begin{array}{r}
\dot{\mathbf{r}}_{\mathbf{i}}=\frac{\mathbf{p}_{\mathbf{i}}}{2 p_{i}^{0}}+\sum_{j}^{N} \frac{m}{p_{j}^{0}} \frac{\partial\left\langle\hat{V}_{j}\right\rangle}{\partial \mathbf{p}_{\mathbf{i}}}, \\
\dot{\mathbf{p}}_{\mathbf{i}}=-\sum_{j}^{N} \frac{m}{p_{j}^{0}} \frac{\partial\left\langle\hat{V}_{j}\right\rangle}{\partial \mathbf{r}_{\mathbf{i}}} . \\
p_{i}^{0}=\sqrt{\mathbf{p}_{\mathbf{i}}^{2}+m^{2}+2 m\left\langle\hat{V}_{i}\right\rangle},
\end{array}
$$

where $\mathbf{r}_{\mathbf{i}}$ is the spatial coordinate of the centroid of the $i$-th nucleon, $\mathbf{p}_{\mathbf{i}}$ is the momentum of the $i$-th nucleon, $m$ is the rest mass of nucleons, $\left\langle\hat{V}_{j}\right\rangle$ is the potential of $j$-th particle, and $N$ is the number of particles in the system. In both JQMD and JQMD-2.0, the potential term of the Hamiltonian $V$ is a sum of the Skyrme-type force term, Coulomb interaction term, and symmetry term. The potential $V_{i}$ is described by the following formula;

$$
V_{i}=\frac{1}{2} \frac{A}{\rho_{\mathrm{s}}}\left\langle\rho_{i}\right\rangle+\frac{1}{1+\tau} \frac{B}{\rho_{\mathrm{s}}^{\tau}}\left\langle\rho_{i}\right\rangle^{\tau}+\frac{1}{2} \sum_{j} \frac{c_{i} c_{j} e^{2}}{\left|\mathbf{R}_{\mathbf{i}}-\mathbf{R}_{\mathbf{j}}\right|} \operatorname{erf}\left(\frac{\left|\mathbf{R}_{\mathbf{i}}-\mathbf{R}_{\mathbf{j}}\right|}{\sqrt{4 L}}\right)+\frac{C_{\mathrm{s}}}{2 \rho_{\mathrm{s}}} \sum_{j}\left(1-2\left|c_{i}-c_{j}\right|\right) \rho_{i j}
$$

where $A$ is a Skyrme force parameter $(=-219.4 \mathrm{MeV}), \rho_{\mathrm{s}}$ is the saturation density $\left(=0.168 \mathrm{fm}^{-3}\right)$, $\left\langle\rho_{i}\right\rangle$ is the overlap integral of wave packets between the $i$-th nucleon and all the other nucleons, $B$ is another Skyrme force parameter $(=165.3 \mathrm{MeV}), \tau$ is $4 / 3, c_{i}$ is 1 for protons and 0 for neutrons, $e$ is the elementary charge, $\mathbf{R}_{\mathbf{i}}$ denotes the position of $i$-th nucleon, $L$ is the square of the width of wave packet representing nucleons $\left(=2 \mathrm{fm}^{2}\right), C_{\mathrm{s}}$ is the symmetry energy parameter $(=25 \mathrm{MeV})$, and $\rho_{i j}$ is the overlap integral of wave functions of the $i$-th and $j$-th nucleons. The potential description of JQMD-2.0 was inherited from previous JQMD without any modifications.

In addition, in-medium effects on nucleon-nucleon scattering cross sections were modified. Previously, the modified version of the Cugnon's formula $[9,10]$, which assumed that scattering is suppressed by the Pauli blocking effect, was always used to calculate the cross sections. In JQMD-2.0, reduced cross sections or free-space cross sections were used in central and peripheral collisions, respectively. Thus in-medium effect on scattering was considered. 


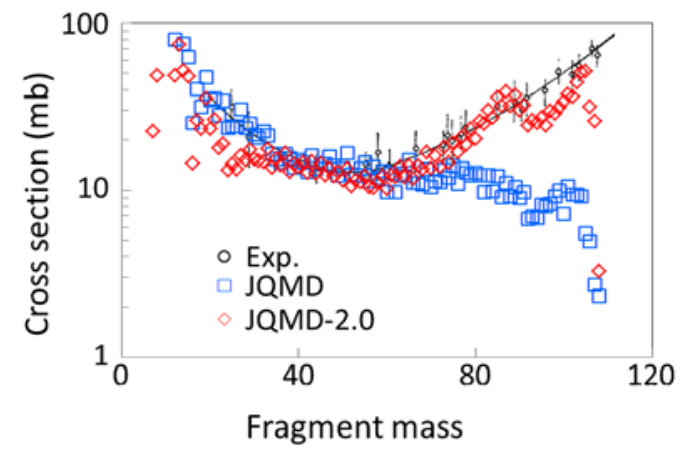

Figure 1. Mass distribution of fragments produced in $3650 \mathrm{MeV} / \mathrm{u}^{\mathrm{Nat}} \mathrm{Ag}\left({ }^{12} \mathrm{C}, \mathrm{x}\right)$ reactions.

After the time evolution of $150 \mathrm{fm} / \mathrm{c}$, nucleons close to each other are bound to finally form clusters. This process was inherited from JQMD without any modifications. Energy balance was checked after the clusterization. Initial energy, which is the sum of projectile kinetic energy, projectile rest mass and target rest mass, was compared with final energy, which is the sum of kinetic energy, excitation energy and the ground-state rest mass of all the clusters. The excitation energies of the clusters were scaled to satisfy the energy conservation. If the energy at the end deviates from that at the beginning so largely that energy cannot be balanced by excitation energy scaling, the simulated reaction event was rejected and event simulation was started afresh.

Thus developed JQMD-2.0 was combined with the statistical decay model GEM (Generalized Evaporation Model) [11] and the statistical multi-fragmentation model (SMM) [12] to calculate fragmentation yields of heavy nuclei such as $\mathrm{Ag}$ and $\mathrm{Au}$.

\section{Results and discussion}

In Fig. 1, mass distribution of the fragments produced in ${ }^{\mathrm{Nat}} \mathrm{Ag}\left({ }^{12} \mathrm{C}, \mathrm{x}\right)$ reaction by $3650 \mathrm{MeV} / \mathrm{u} \mathrm{C}$ ions was calculated and compared with the measurement data [13]. Yields above $\mathrm{A}=70$ was underestimated by JQMD but the simulation by JQMD-2.0 is in better agreement with the measurement data because the improvement in this study is particularly effective for peripheral collisions. It is also reasonable that the calculation by JQMD and that by JQMD-2.0 are mostly the same in the range from $\mathrm{A}=35$ to $\mathrm{A}=70$ because these nuclei are produced by intermediate collisions. The measured mass distribution exhibits steady declining trend down to $\mathrm{A}=65$ whereas the yield calculated by JQMD-2.0 has a dip near $A=95$. The cause of this dip is discussed later.

Fig. 2 shows the comparison of calculated and measured [13] mass distribution of fragments produced in ${ }^{197} \mathrm{Au}\left({ }^{12} \mathrm{C}, \mathrm{x}\right)$ reaction by $3650 \mathrm{MeV} / \mathrm{u} \mathrm{C}$ ions. The cross section is increased in a wider mass range in Fig. 2 because $\mathrm{Au}$ has a larger nuclear surface than $\mathrm{Ag}$ and the improvement in this study is particularly effective in peripheral collisions. Similar to the case of Ag, the mass distribution calculated by JQMD and JQMD-2.0 has a dip aroud $\mathrm{A}=170$, which is not seen in the measurement data.

To clarify the cause of the dip in the calculated mass distribution, fragment mass distributions for ${ }^{\text {Nat }} \mathrm{Ag}\left({ }^{12} \mathrm{C}, \mathrm{x}\right)$ reaction were calculated with changing the statistical decay model. The comparison is shown in Fig.3, 


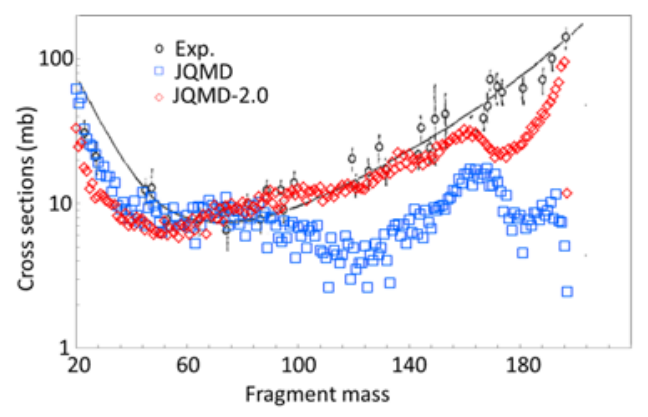

Figure 2. Mass distribution of fragments produced in $3650 \mathrm{MeV} / \mathrm{u}{ }^{197} \mathrm{Au}\left({ }^{12} \mathrm{C}, \mathrm{x}\right)$ reactions.

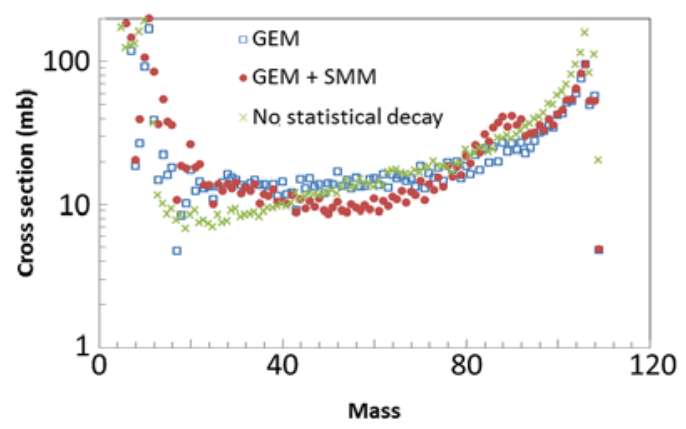

Figure 3. Comparison of fragment mass distribution in $3650 \mathrm{MeV} / \mathrm{u}^{\mathrm{Nat}} \mathrm{Ag}\left({ }^{12} \mathrm{C}, \mathrm{x}\right)$ reactions.

The dip was not observed if SMM was turned off though the yields below $A=20$ become insufficient. The comparison of GEM+SMM, GEM, and no statistical decay (i.e., just after JQMD) suggests that the yield bump at the left of the dip is attributed to the statistical decay and particularly to the SMM stage. It may be explained by the asymmetric binary decay in SMM, which takes place if the pre-fragment has excitation energy about $4 \mathrm{MeV} /$ nucleon [14]. Both GEM and GEM+SMM underestimate the yield above $\mathrm{A}=90$; therefore nucleon knock-out and residual nucleus exitation in peripheral collisions should be suppressed in JQMD-2.0 for more precise prediction.

\section{Conclusion}

It is shown that mass distribution of the fragments produced by interactions of heavy nuclei such as $\mathrm{Au}$ and Ag with heavy ions was predicted by JQMD-2.0 better than the old JQMD. The yield of fragment with mass close to the original mass was significantly improved. Particularly the improvement was pronounced for Au than for Ag because of larger cross sections corresponding to peripheral collisions.

The fragmentation of heavy nuclei is significantly affected by multifragmentation, dip of yield attributed to multifragmentation is observed only in calculated result. This fact indicates that revision of reaction mechanism description relevant to estimate of nucleon loss and excitation energies in peripheral collisions is the key for more accurate prediction of fragment yield. 
JQMD-2.0 is incorporated to PHITS Version 2.76 and later and is generally applicable for calculation of residual nuclide production and secondary particle production.

\section{Acknowledgments}

We wish to thank Dr. Davide Mancusi for providing the source code of R-JQMD. We also thank the operation team of the Center for Computational Science and E-systems (CCSE) in the Japan Atomic Energy Agency. Monte-Carlo simulations reported in this paper were executed on a PC-cluster system of CCSE. This work was partly supported by JSPS KAKENHI Grant Numbers 24860072 (Grant-inAid for Research Activity Start-up), and 26790072 (Grant-in-Aid for Young Scientists B). 


\section{References}

[1] K. Niita, S. Chiba, T. Maruyama, T. Maruyama, H. Takada, T. Fukahori, Y. Nakahara, A. Iwamoto, Phys. Rev. C 52, 2620 (1995)

[2] T. Sato, K. Niita, N. Matsuda, S. Hashimoto, Y. Iwamoto, S. Noda, T. Ogawa, H. Iwase, H. Nakashima, T. Fukahori et al., Journal of Nuclear Science and Technology 50, 913 (2013), http://dx.doi.org/10.1080/00223131.2013.814553

[3] D. Satoh, T. Kurosawa, T. Sato, A. Endo, M. Takada, H. Iwase, T. Nakamura, K. Niita, Nuclear Instruments and Methods in Physics Research Section A: Accelerators, Spectrometers, Detectors and Associated Equipment 583, 507 (2007)

[4] D. Satoh, D. Moriguchi, T. Kajimoto, Y. Koba, Y. Nakamura, N. Shigyo, M. Ueyama, U. Y., Y. M., J. Korean Phys. Soc. 59, 1741 (2011)

[5] T. Ogawa, T. Sato, S. Hashimoto, D. Satoh, S. Tsuda, K. Niita, Phys. Rev. C 92, 024614 (2015)

[6] T. Ogawa, T. Sato, S. Hashimoto, K. Niita, journal of italian physical society to be published, (2016)

[7] T. Ogawa, T. Sato, S. Hashimoto, K. Niita, CERN Conference series to be published, (2016)

[8] D. Mancusi, K. Niita, T. Maruyama, L. Sihver, Phys. Rev. C 79, 014614 (2009)

[9] J. Cugnon, T. Mizutani, J. Vandermeulen, Nuclear Physics A 352, 505 (1981)

[10] J. Cugnon, Phys. Rev. C 22, 1885 (1980)

[11] S. Furihata, Nuclear Instruments and Methods in Physics Research Section B: Beam Interactions with Materials and Atoms 171, 251 (2000)

[12] T. Ogawa, T. Sato, S. Hashimoto, K. Niita, Nuclear Instruments and Methods in Physics Research Section A: Accelerators, Spectrometers, Detectors and Associated Equipment 723, 36 (2013)

[13] P. Kozma, C. Damdinsuren, Czechoslovak Journal of Physics 40, 38 (1990)

[14] J. Bondorf, R. Donangelo, I. Mishustin, H. Schulz, Nuclear Physics A 444, 460 (1985) 